The LNCS series reports state-of-the-art results in computer science research, development, and education, at a high level and in both printed and electronic form. Enjoying tight cooperation with the R\&D community, with numerous individuals, as well as with prestigious organizations and societies, LNCS has grown into the most comprehensive computer science research forum available.

The scope of LNCS, including its subseries LNAI and LNBI, spans the whole range of computer science and information technology including interdisciplinary topics in a variety of application fields. The type of material published traditionally includes

- proceedings (published in time for the respective conference)

- post-proceedings (consisting of thoroughly revised final full papers) - research monographs (which may be based on outstanding PhD work, research projects, technical reports, etc.)

More recently, several color-cover sublines have been added featuring, beyond a collection of papers, various added-value components; these sublines include

- tutorials (textbook-like monographs or collections of lectures given at advanced courses)

state-of-the-art surveys (offering complete and mediated coverage of a topic)

hot topics (introducing emergent topics to the broader community) In parallel to the printed book, each new volume is published electronically in LNCS Online.

Detailed information on LNCS can be found at

www.springer.com/lncs

Proposals for publication should be sent to

LNCS Editorial, Tiergartenstr. 17, 69121 Heidelberg, Germany

E-mail: lncs@springer.com

ISSN 0302-9743

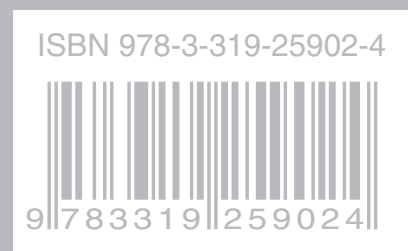

Lecture Notes int Computer Science LNNCS - LNAI - LNB
Sebastiano Battiato - Jacques Blanc-Talon

Giovanni Gallo $\cdot$ Wilfried Philips

Dan Popescu $\cdot$ Paul Scheunders (Eds.)

\section{Advanced Concepts} 品 for Intelligent 里 兴 Vision Systems

16th International Conference, ACIVS 2015

Catania, Italy, October 26-29, 2015

Proceedings 